\title{
Transatlantica
}

Revue d'études américaines. American Studies Journal

Cartographies de l'Amérique / Histoires d'esclaves

\section{Histoires d'esclaves}

\section{Nathalie Dessens et Jean-Pierre Le Glaunec}

\section{(2) OpenEdition}

\section{Journals}

Édition électronique

URL : https://journals.openedition.org/transatlantica/6235

DOI : $10.4000 /$ transatlantica.6235

ISSN : 1765-2766

Éditeur

Association française d'Etudes Américaines (AFEA)

Référence électronique

Nathalie Dessens et Jean-Pierre Le Glaunec, «Histoires d'esclaves », Transatlantica [En ligne], 2 | 2012, mis en ligne le 20 mai 2013, consulté le 06 avril 2023. URL : http://journals.openedition.org/ transatlantica/6235; DOI : https://doi.org/10.4000/transatlantica.6235

Ce document a été généré automatiquement le 6 avril 2023.

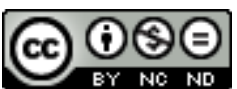

Creative Commons - Attribution - Pas d'Utilisation Commerciale - Pas de Modification 4.0 International - CC BY-NC-ND 4.0

https://creativecommons.org/licenses/by-nc-nd/4.0/ 


\title{
Histoires d'esclaves
}

\author{
Nathalie Dessens et Jean-Pierre Le Glaunec
}

1 Quatre siècles d'esclavage aux Amériques et des millions d'esclaves déracinés de leur Afrique natale ou nés dans les Amériques. Des millions d'esclaves et autant d'histoires. Des histoires de vie, de résistance et d'adaptation, de solidarité et de solitude, de violence et de lutte, du Brésil à la Nouvelle-France en passant par la Caraïbe, et bien sûr, les États-Unis.

2 Longtemps, l'histoire de l'esclavage s'est écrite sans que l'on entende vraiment la voix, ou les voix, des esclaves. La plupart des sources ne se prêtent pas, il est vrai, ou du moins en apparence, à une histoire de voix. Les archives judiciaires font entendre généralement des voix d'esclaves rapportées et déformées, comme le rappelle Ada Ferrer dans le cas de Cuba: «the words of the enslaved are recorded by scribes who paraphrase them in the third person, thus intervening in what was recorded, remembered, and now available to us as scholars and students $»^{1}$. Les livres de plantations et les inventaires après décès égrènent des listes de noms auxquels sont associés parfois un âge, parfois un prix ou encore un métier - tel esclave est dit tonnelier, tel autre est propre aux travaux des champs. Les esclaves mentionnés dans ces documents créés par les maitres ne parlent pas, pas plus que les autres biens - outils agricoles, animaux et autres bâtiments - qu'ils côtoient sur le papier. Les annonces de vente ou de fuite d'esclaves publiées chaque jour dans les gazettes des sociétés esclavagistes évoquent, certes, des fragments de vie de plusieurs dizaines de milliers d'esclaves mais elles permettent surtout de reconstituer l'histoire de sociétés blanches, des sociétés de terreur où s'inventait chaque jour un pouvoir qui s'appuyait sur un dogme racial en perpétuelle construction et redéfinition. Le problème auquel les historiens de l'esclavage - et les historiens des sans voix en général - sont confrontés est bien connu. L'auteure Mimi Sheller le résume ainsi: "Seldom are there "direct" sources of writing from a largely illiterate population; much of what we know is based on records kept by others, second or third-hand stories or oral histories and transcribed narratives $»^{2}$.

3 Hormis de rares exceptions ${ }^{3}$, l'histoire de l'esclavage aux États-Unis avant les années 1960 a représenté des femmes et des hommes sans voix et sans histoire. Les sources 
disponibles - sources judiciaires, journaux, livres de plantation etc. - ne semblaient vouloir / devoir parler. Ce n'est que dans la foulée du mouvement pour les droits civiques que des premières vraies tentatives ont été réalisées pour réparer l'oubli, pour combler les silences, pour reconnaitre aux esclaves une capacité d'action qui leur était propre. Les esclaves avaient bien une voix et cette voix pouvait être entendue. En effet, comme le formule Karina Williamson, auteure de Contrary Voices. Representations of West Indian Slavery, 1657-1834, "the voices of the enslaved nevertheless survive through a range of channels, including published transcriptions of songs, folk-tales, proverbs, funeral orations, reported conversations and witness statements $»^{4}$. Aux États-Unis, des historiens se mirent à re/constituer des histoires d'esclaves, par le bas cette fois, «from the bottom up ". Ils utilisèrent pour cela les « récits esclaves " publiés au XIX ${ }^{e}$ siècle dans le cadre du mouvement abolitionniste et les entretiens menés auprès d'anciens esclaves à la fin des années 1930 dans le cadre de la Works Progress Administration. Ces sources jouèrent un rôle important dans la mise en place de la nouvelle histoire sociale de l'esclavage dans la mesure où elles permirent de donner aux asservis de nouveaux visages, de nouveaux sentiments et de nouvelles émotions que l'histoire n'avait, jusquelà, pas envisagés.

4 Si de nombreux historiens ont, depuis, utilisé ces deux types de sources, les articles de ce dossier montrent qu'elles recèlent encore bien des trésors. Suzanne Fraysse et Dominique Aurélia démontrent combien les récits d'esclaves du XIX ${ }^{e}$ siècle, en dépit de la violence, sexuelle notamment, dont ils témoignaient, ont permis de rendre aux femmes et aux hommes asservis leur humanité, comment leur écriture, dont Suzanne Fraysse explique qu'elle s'inscrit dans une " culture du sentiment et de la compassion ", était, pour les esclaves, une façon de revendiquer leur liberté et d'affirmer leur capacité de résistance et leur droit à l'intimité (voir l'article de S. Fraysse), mais aussi, pour les femmes esclaves, leur féminité. Ce dernier aspect est développé par Dominique Aurélia dans un article qui étudie la voix, marginale par rapport à celle, mieux connue, des narrateurs esclaves masculins, de trois femmes, Mattie Jackson, Kate Drumgoold et Annie Burton. Les entretiens de la WPA sont, eux aussi, des sources inépuisables pour reconstituer des histoires d'esclaves, comme le montre bien l'article de Lori Lee, qui n'oublie pas de rappeler les précautions qu'il convient de prendre pour en faire l'analyse, en particulier des relations de pouvoir qui en sous-tendent la construction. Loin d'être obsolètes, ces entretiens ont encore beaucoup à nous apprendre, comme l'a également montré, par exemple, Stephanie Camp dans son étude récente des résistances quotidiennes des esclaves dans le Sud états-unien, Closer to Freedom. Enslaved Women and Everyday Resistance in the Plantation South ${ }^{5}$.

5 Si les récits publiés ou enregistrés ont été les premiers à être utilisés pour raconter des histoires d'esclaves, ils n'ont été que le premier pas vers une connaissance approfondie des itinéraires individuels et parfois collectifs des Amériques serviles. Déplorant la réduction que produisait la médiation politisée de la voix des esclaves dans les récits ou les enquêtes orales, les historiens se sont également tournés vers d'autres sources - judiciaires, religieuses, notariales, épistolaires etc. - pour dessiner le portrait des communautés d'esclaves. Dans son article, Anne-Claire Faucquez utilise des recensements, des actes de ventes et des archives judiciaires pour mettre en question un certain nombre d'idées reçues sur l'esclavage urbain à New-York dans la deuxième moitié du XVII siècle et pour démontrer l'influence particulière d'un environnement donné sur l'esclavage et sur la vie des esclaves qui y résidaient. Elizabeth Neidenbach, 
quant à elle, interprète deux testaments écrits par Marie Justine Cirnaire, dite Veuve Couvent, à la Nouvelle-Orléans, en 1812 et 1832. Grâce à ces deux textes, elle nous raconte l'histoire d'une femme exceptionnelle, née en Afrique, réduite en esclavage, puis libérée, devenue propriétaire, gestionnaire et testataire d'une fortune conséquente. Mais ce que lit Elizabeth Neidenbach dans l'histoire de Mme Couvent, c'est aussi les évolutions que traduisent les deux versions du document et ce qu'elles nous apprennent sur le contexte de la vie de leur auteur, révélant les spécificités légales, sociales et culturelles de la Nouvelle-Orléans esclavagiste.

Si Mme Couvent a quitté la colonie de Saint-Domingue dans la foulée de la Révolution des esclaves de 1791, c'est à Saint-Domingue que se déroule l'histoire, ou plutôt les histoires que nous raconte Jean-Louis Donnadieu, celles de Jean-Jacques et Hippolyte, deux commandeurs meneurs de grèves. Si l'article décentre un peu le point de vue vers la Caraïbe francophone, il permet d'ouvrir le dossier à un espace atlantique fertile en itinéraires, en rencontres d'hommes et d'idées, en échanges et en mobilités.

7 Les articles de ce dossier, de Suzanne Fraysse à Jean-Louis Donnadieu, couvrent des espaces géographiques vastes et puisent à des méthodologies diverses. Ils nous racontent tous, chacun à leur manière, des histoires d'esclaves. L'on y rencontre Lysbet Anthony, Elizabeth, Marramita, Marie-Justine Cirnaire, Fountain Hughes, Laura Smalley, Jean-Jacques et Hippolythe, Furcy, Frederick Douglass, Louisa Piquet, Mattie Jackson, Kate Drumgoold ou encore Annie Burton. Les articles de ce dossier nous permettent également de mieux comprendre comment retracer des histoires d'esclaves et nous apprennent comment écouter des voix dont l'écho subsiste à peine. Ils retracent des itinéraires individuels mais proposent également une réflexion théorique et méthodologique sur la reconstitution de ces itinéraires. À ce titre, ils répondent tous, au bout du compte, à la même question: comment écrire l'histoire de femmes et d'hommes à qui l'on a refusé pendant longtemps la possibilité et le droit d'écrire leur propre histoire?

Cette question est au cœur de l'article de Susan Peabody qui ouvre le dossier, et par lequel nous aimerions paradoxalement clore cette présentation. L'exemple qu'elle prend se situe dans l'Océan Indien mais sa réflexion résume la problématique qui recoupe toutes les contributions présentées ici. Analysant les différents moyens qui s'offrent à l'historien pour écrire des histoires d'esclaves, l'auteure compare la microhistoire, la biographie et même la fiction, et elle propose une réflexion passionnante sur la façon de transformer des sources forcément lacunaires en autant de récits de vie, comme en témoignent les trois versions de la même histoire qu'elle nous propose en annexe de son article.

9 Pour conclure cette introduction, il est tentant d'emprunter les mots d'ouverture d'une autre introduction, celle qui inaugure le volume d'essais dirigé par Stephanie Camp et Edward Baptist, New Studies in the History of American Slavery, publié en 2006:

This volume represents one more step in a long process of trying to understand slavery, a process that actually began aboard the slave ships: the enslaved themselves were the first people to tell themselves and others what was happening in West and Central Africa, along the Middle Passage, and in the slave markets of the Americas. From the sixteenth through the twentieth centuries, the slaves were the first to formulate histories of enslavement ${ }^{6}$.

10 Comme le rappellent Stephanie Camp et Edward Baptist, les esclaves furent les premiers à raconter, et à se raconter, des / leurs histoires. Les articles de ce dossier reposent tous sur ce constat, simple en apparence, mais qui évoque au fond le principal 
défi des spécialistes de l'esclavage, des États-Unis et des autres sociétés esclavagistes de la période moderne : celui de retrouver et de faire entendre des histoires d'esclaves qui n'ont jamais été sans voix.

\section{NOTES}

1. Avant-propos de Ada Ferrer dans Gloria García Rodríguez, Voices of the Enslaved in NineteenthCentury Cuba, Chapel Hill, The University of North Carolina Press, 2011, xiii.

2. Mimi Sheller, Democracy After Slavery: Black Publics and Peasant Radicalism in Haiti and Jamaica, Basingstoke, Macmillan Education, 2000, 199.

3. Voir les travaux de W. E. B. Du Bois, C. L. R. James, Herbert Aptheker ou encore John Hope Franklin.

4. Karina Williamson, dir., Contrary Voices. Representations of West Indian Slavery, 1657-1834, Kingston, University of the West Indies Press, 2008, 4.

5. Stephanie Camp, Closer to Freedom. Enslaved Women and Everyday Resistance in the Plantation South, Chapel Hill et Londres, The University of North Carolina Press, 2004. Sur l'utilisation des entretiens de la WPA, voir page 8.

6. Edward Baptist et Stephanie Camp, eds., New Studies in the History of American Slavery, Athens et Londres, The University of Georgia Press, 2006, 1. 